\title{
The Mediterranean way: why elderly people should eat wholewheat sourdough bread-a little known component of the Mediterranean diet and healthy food for elderly adults
}

\author{
Antonio Capurso ${ }^{1} \cdot$ Cristiano Capurso $^{2}$
}

Received: 10 August 2019 / Accepted: 16 October 2019 / Published online: 13 November 2019

(c) Springer Nature Switzerland AG 2019

\section{Background}

Bread has been a staple food of Mediterranean populations since the time of the Greek and Roman civilizations. When pasta was later introduced, the average total daily caloric intake from carbohydrates reached almost $50 \%$ of the diets of the peoples living in Southern Italy and Greece, populations known to be at low risk of coronary heart disease (CHD) [1, 2].

In the early 1960s, when Southern Italian and Greek cohorts were enrolled in the "Seven Countries Study", bread was still a staple food in standard diets and one of the most important sources of calories. When the dietary habits of Southern Mediterranean populations began the transition to western standards beginning in the 1960s, the process tended to proceed at a slower rate in the poor rural areas of the Southern Mediterranean area with respect to the richer ones in north/west Europe and in the lower social classes with respect to higher ones.

The bread the rural Southern European populations traditionally consumed was brown and made from whole wheat flour, often mixed with barley, ground in stone mills and prepared using a homemade spontaneous sourdough starter and not a commercial agent. Traditionally, sourdough bread refers to bread leavened using a sourdough starter (a natural leaven), which is a mixture of flour and water inhabited by so-called wild yeasts and bacteria that cause the dough to rise and give it its distinguishing flavor. During fermentation a lactic acid bacteria and yeast ecosystem is created. Refreshing the starter almost daily by adding new flour and

Antonio Capurso

a.capurso@alice.it

1 Department of Internal Medicine, School of Medicine, University of Bari "Aldo Moro", Bari, Italy

2 Department of Medical and Surgical Science, School of Medicine, University of Foggia, Foggia, Italy water to the mixture keeps the microorganisms in an active state. Yeasts, which are the primary producers of $\mathrm{CO}_{2}$ in sourdough, are considered responsible for the leavening process. Both homo-fermentative and hetero-fermentative lactic acid bacteria are responsible for dough acidification: the former produces lactic acid and the latter lactic acid plus $\mathrm{CO}_{2}$, acetic acid, and/or ethanol [3].

Habitual consumption of wholemeal sourdough bread has contributed to reducing the risk of coronary heart disease (CHD), diabetes and cancer in southern Mediterranean populations.

\section{Sourdough fermentation, the glycemic index (GI) and the glycemic load (GL)}

The glycemic index (GI) is the number from 0 to 100 assigned to a food (pure glucose has been arbitrarily given the value of 100) which is indicative of the relative rise in blood glucose levels found $2 \mathrm{~h}$ after the food has been consumed. The GI of a specific food depends primarily on the quantity and type of carbohydrate it contains, but it is also affected by numerous other factors including the amount of organic acids.

The glycemic load (GL) is a value indicating how quickly a given food portion elevates blood glucose levels. It takes into account both the amount of carbohydrates in the serving and how quickly it raises blood glucose levels $(\mathrm{GL}=\mathrm{GI} \times$ carbohydrate/100). A GL of $0-10=$ low GL; 11-19=medium GL; 20 and over = high GL).

Sourdough fermentation of wheat flour dough significantly lowers the GI of bread by reducing the rate of starch digestion, mostly through the formation of organic acids that delay the absorption of starch [4, 5]. Starch is absorbed more slowly in the presence of lactic acid due to the inhibition of amylolytic enzymes [6], and its bioavailability is reduced 
due to the interaction between starch and gluten [7]. Acetic acid delays the gastric empting rate [8].

Here are some examples of glycemic loads and indexes. The GI for a $30 \mathrm{~g}$ serving of white wheat flower bread is 71 and its GL is 9 [8]. The GI for a $30 \mathrm{~g}$ serving of whole wheat flour bread is 71, and its GL is 10 [8]. The GI of a 30 $\mathrm{g}$ serving of sourdough wheat bread is 54 and its GL is 8 [8]. According to the 2002 edition of the international tables of GI Index and GL values [9], $<55$ is defined as low, between 56 and 69 is defined as moderate, and $>70$ is considered high. Sourdough wheat bread thus falls into a low GI food category level.

One of the components of the traditional Mediterranean diet, wholemeal sourdough bread is part of a low GI diet. Low GI/GL diets offer many health benefits especially for patients with type 2 diabetes mellitus (T2DM), CHD and cancer and for subjects who are overweight or hyperlipidemic.

\section{Sourdough bread and type 2 diabetes mellitus}

While whole wheat and white breads elicit the same postprandial response of glucose and insulin in T2DM patients [10], the inclusion of a high percentage of intact or partially milled cereal kernels in the flour reduces the bread's glycemic response [11-13]. The effect seems to be due to the fact that wheat germ contains a natural amylase inhibitor that is destroyed when it passes through a roller mill. Standard wholemeal flour (not stone ground meal flour) is a reconstituted flour after it has passed through the roller mill which means that whole meal flour is hydrolyzed at the same identical rate as white flour.

When four breads prepared from two wheat flours (white and whole) leavened through two processes (sourdough and Saccharomyces cerevisiae) were fed to eight healthy volunteers, it was found that both sourdough fermented breads showed a significantly lower glycemic response with respect to the breads leavened with $S$. cerevisiae. The presence of fiber did not seem to influence the breads' glycemic potential [14].

In another study, 16 glucose-intolerant subjects were randomly fed a meal containing bread $(70 \%$ durum wheat semolina and $30 \%$ corn flour) leavened with sourdough or a meal containing bread leavened with baker's yeast. The former induced a significantly lower plasma glucose response at $30 \mathrm{~min}$ and a significantly smaller incremental area under the curve at 0-30 and 0-60 min with respect to the latter. Likewise, the plasma insulin response of the former showed significantly lower values at $30 \mathrm{~min}$ and a significantly smaller incremental area under the curve at 0-30 $\min$ [15].
The investigators of another study reported that out of four breads being tested (white wheat bread, wholemeal wheat bread, sourdough wholemeal bread and wholemeal bread made with xylanase) the sourdough wholemeal wheat bread was associated to the lowest postprandial glucose and insulin response [16].

In a meta-analysis including 4 prospective cohort studies, with a GL ranging from $\sim 60$ to $\sim 280$ g per daily intake of $2000 \mathrm{kcal}$, the GL was positively associated with RR of T2DM of 1.45 (95\% CI 1.31, 1.61) for a 100-g increment in GL $(P<0.001)[17]$.

High GL diets can increase the risk of T2DM over a period of years by increasing insulin demand following hyperglycemia which, in turn, affects pancreatic function (due to $\beta$-cell exhaustion or toxicity of hyperglycemia) or because of a higher insulin resistance induced by free fatty acids produced in the late postprandial period by counterregulatory hormones. The progressively higher glucose levels induced by a high GL depend on the underlying insulin resistance which is more evident in obese, inactive or genetically susceptible individuals [18]. The mechanisms by which $\mathrm{Gl}$, hyperinsulinemia, and insulin resistance lead to T2DM seem to be quite complex and heavily affected by an increase in abdominal-perivisceral fat [19].

\section{Sourdough leavened bread and body weight}

Diets with a high GI tend to elevate glucose and insulin in the early postprandial period ( $0-2 \mathrm{~h}$ ), and as insulin excess quickly lowers blood glucose in the postprandial period (3-5 h), appetite is increased. A decrease in plasma glucose and greater hunger in overweight or obese men have been associated with increased activity in brain regions related to food intake, reward and craving. Limbic-striatal brain activation with a concomitant enhanced desire for high-calorie foods was noted when a mild hypoglycemia occurred during hyperinsulinemic euglycemic-hypoglycemic clamp by a study examining 14 healthy subjects who underwent arterial spin-labeling functional magnetic resonance imaging [20, 21].

A meta-analysis evaluating the relationship between glycemic response and markers of health demonstrated that low GL diets were significantly associated with lower body weight in free living subjects if a GL was reduced by $\geq 17 \mathrm{~g}$ glucose equivalent/day. The body weight reduction was even greater when the GL was reduced by $>42 \mathrm{~g}$ glucose equivalent/day [22]. Although some observational studies have shown conflicting results with regard to the relationship between GI/GL and body mass index [23-26], most findings suggest that sourdough-leavened wholemeal bread significantly contributes to reducing excess body weight because of its low GI/GL index. 


\section{Sourdough leavened bread, serum lipid profile and risk of coronary heart disease (CHD)}

Sourdough wholemeal bread has a beneficial effect on serum lipid profiles and CHD risk factors. Large cohort studies have shown that GL is inversely associated with HDL-cholesterol concentrations and directly associated with triglyceride levels [27-30]. In 5830 non-diabetic subjects between the ages of 20 and 70 participating in the Health Worker Cohort Study, the adjusted odds ratios in the highest versus the lowest quartile of dietary GL were 1.78 for low HDL-cholesterol levels $(<40 \mathrm{mg} / \mathrm{dL}$ for men; $<50 \mathrm{mg} / \mathrm{dL}$ for women) and 1.85 for high triglyceride levels ( $\geq 150 \mathrm{mg} / \mathrm{dL}$ ) [28]. The highest HDLcholesterol concentration and the lowest triglyceride and insulin levels were observed in the lowest GI tertile by a cross-sectional study examining dietary GI/GL based on three-day dietary records [31].

An increase in insulin resistance which in turn causes an increase in triglycerides and a fall in HDL-cholesterol can explain the effects of diets with a high GI/GL index on blood lipids. In the early postprandial period $(0-2 \mathrm{~h}$ after the meal), the rapid absorption of carbohydrates after a high GI meal leads to a relatively high blood glucose level and a high insulin/glucagon ratio. In the late postprandial period (4-6 $\mathrm{h}$ after the meal), the counter-regulatory hormones restore normal glycemia but cause a marked increase in free fatty acid plasma concentrations [32]. Elevated levels of glucose, insulin and free fatty acids induce insulin resistance, an important CHD risk factor [33].

Interestingly, the hazards ratio for CHD has been found to be higher in diabetic women than in men [34]. In fact, the investigators of the EPICOR (long-term follow-up of antithrombotic management Patterns In acute CORonary syndrome patients) study found that in women a high carbohydrate intake of foods with a high GI index but not of foods with a low one was significantly associated with greater risk of CHD [35]. Dietary GI can affect the risk of CHD also via inflammation and oxidative stress mechanisms. Low GI diets are also associated with lower C-reactive protein [29] and lipid peroxidation markers [36].

\section{GI, GL and cancer development}

Foods with high GI and GL indexes may favour cancer development. Chronic hyperinsulinemia, often associated with frequent intake of foods with a high GI and GL index, promote cancer development through stimulation of multiple cellular signaling cascades and increasing the bioactivity of insulin-like growth factor-1. As far as breast cancer is concerned, insulin and IGF-I inhibit the hepatic synthesis of sex hormone binding insulin (SHBG). The bioavailability of estrogen is increased thus promoting cellular proliferation and inhibiting apoptosis in breast epithelium and endometrium [37]. Moreover, hyperinsulinemia increases proinflammatory cytokines and oxidative stress which can, in turn, promote malignancy and neoplastic progression [37]. A large nationwide populationbased case-control study demonstrated that GI was positively associated with the risk of prostate cancer, while a high GL significantly increased the risk of colorectal and pancreatic cancers [38].

\section{The fiber in sourdough-leavened wholemeal bread}

It is important to remember that wholemeal sourdoughleavened bread is rich in fiber. High intake of cereal fiber reduces the risk of CHD [39], T2DM [40], obesity [41] and colorectal cancer [42]. Cereal fiber also reduces inflammatory markers [43, 44] and improves insulin sensitivity [45]. Whole grain cereals, but not refined grains, reduce the risk of CHD, gastrointestinal cancer [46] and T2DM [46, 47].

The protective mechanisms triggered by whole grain cereals against the risk of developing chronic diseases depend on the bioactive compounds and dietary fiber contained in the bran and germ fractions that are lost in the milling process [48]. Interestingly, fiber and oligosaccharides of whole grain wheat also have a prebiotic effect on the gut microbiota [49, 50] causing systemic low-grade inflammation and the progression of chronic metabolic diseases [51].

\section{Conclusions}

Sourdough bread has been considered a healthy food since as far back as ancient times. The wheat used is ground in stone and not in roller mills that destroy some of the nutrients in wheat germ (vitamins, minerals and enzymes). It is made using a sourdough starter composed of natural yeast and lactobacilli (beneficial bacteria). Left to rise for 7-8 $\mathrm{h}$ and then baked, the bread contains all the nutrients present in whole grains and, in particular, those in wheat germ. In addition to high-quality proteins and essential fatty acids, it contains a wealth of vitamins and minerals. Sourdough bread, in fact, provides vitamin-E, vitamin-B1, B6, B12, thiamin, niacin, folate, riboflavin, potassium, zinc, iron, magnesium, selenium, calcium, phosphorus and manganese. The bread's numerous beneficial effects are for the most part linked to two of its peculiar characteristics: the sourdough starter and wheat germ. 
Acknowledgements The authors acknowledge the assistance of Ms Linda Inverso in editing the manuscript. This paper has been endorsed by the "Fondazione Dieta Mediterranea, Ostuni, Italy".

\section{Compliance with ethical standards}

The authors state that accepted principles of ethical and professional conduct have been followed.

Conflict of interest The authors also state that there is no conflict of interest.

Statement of human and animal rights This paper neither involves human participants nor animals.

Informed consent For this reason, neither ethical approval nor informed consent was requested.

\section{References}

1. Alberti-Fidanza A, Fidanza F, Chiuchiù MP et al (1999) Dietary studies on two rural italian population groups of the Seven Countries Study. 3. Trend of food and nutrient intake from 1960 to 1991. Eur J Clin Nutr 53:854-860

2. Simopoulos AP (2001) The Mediterranean diets: what is so special about the diet of Greece? The scientific evidence. J Nutr 131:3065-3073

3. Corsetti A, Settanni L (2007) Lactobacilli in sourdough fermentation. Food Res Int 40:539-558

4. Björck I, Elmståhl HL (2003) The glycaemic index: importance of dietary fibre and other food properties. Proc Nutr Soc 62:201-206

5. Poutanen K, Flander L, Katina K (2009) Sourdough and cereal fermentation in a nutritional perspective. Food Microbiol 26:693-699

6. Liljeberg HG, Lönner CH, Björck I (1995) Sourdough fermentation or addition of organic acids or corresponding salts to bread improves nutritional properties of starch in healthy humans. J Nutr 125:1503-1511

7. Liljeberg H, Björck I (1998) Delayed gastric emptying rate may explain improved glycaemia in healthy subjects to a starchy meal with added vinegar. Eur J Clin Nutr 52:368-371

8. Atkinson FS, Foster-Powell K, Brand-Miller JC (2008) International tables of glycemic index and glycemic load values: 2008. Diabetes Care 31:2281-2283

9. Foster-Powell K, Holt SH, Brand-Miller JC (2002) International table of glycemic index and glycemic load values: 2002. Am J Clin Nutr 76:5-56

10. Breen C, Ryan M, Gibney MJ et al (2013) Glycemic, insulinemic, and appetite responses of patients with type 2 diabetes to commonly consumed breads. Diabetes Educ 39:376-386

11. Jenkins DJ, Wesson V, Wolever TM et al (1988) Wholemeal versus wholegrain breads: proportion of whole or cracked grain and the glycaemic response. BMJ 297:958-960

12. Holt SH, Miller JB (1994) Particle size, satiety and the glycaemic response. Eur J Clin Nutr 48:496-502

13. Liljeberg H, Granfeldt Y, Björck I (1992) Metabolic responses to starch in bread containing intact kernels versus milled flour. Eur J Clin Nutr 46:561-575

14. Scazzina F, Del Rio D, Pellegrini N et al (2009) Sourdough bread: starch digestibility and postprandial glycemic response. J Cereal Sci 49:419-421
15. Maioli M, Pes GM, Sanna M et al (2008) Sourdough leavened bread improves postprandial glucose and insulin plasma levels in subjects with impaired glucose tolerance. Acta Diabetol 45:91-96

16. Lappi J, Selinheimo E, Schwab U et al (2010) Sourdough fermentation of wholemeal wheat bread increases solubility of arabinoxylan and protein and decreases postprandial glucose and insulin responses. J Cereal Sci 51:152-158

17. Livesey G, Taylor R, Livesey $\mathrm{H}$ et al (2013) Is there a doseresponse relation of dietary glycemic load to risk of type 2 diabetes? Meta-analysis of prospective cohort studies. Am J Clin Nutr 97:584-596

18. Willett W, Manson J, Liu S (2002) Glycemic index, glycemic load, and risk of type 2 diabetes. Am J Clin Nutr 76:274-280

19. Capurso C, Capurso A (2012) From excess adiposity to insulin resistance: the role of free fatty acids. Vasc Pharmacol 57:91-97

20. Lennerz BS, Alsop DC, Holsen LM et al (2013) Effects of dietary glycemic index on brain regions related to reward and craving in men. Am J Clin Nutr 98:641-647

21. Page KA, Seo D, Belfort-DeAguiar R et al (2011) Circulating glucose levels modulate neural control of desire for high-calorie foods in humans. J Clin Investig 121:4161-4169

22. Livesey G, Taylor R, Hulshof T et al (2008) Glycemic response and health. A systematic review and meta-analysis: relations between dietary glycemic properties and health outcomes. Am J Clin Nutr 87:258-268

23. Lau C, Toft U, Tetens I et al (2006) Association between dietary glycemic index, glycemic load, and body mass index in the Inter99 study: Is underreporting a problem? Am J Clin Nutr 84:641-645

24. Murakami K, McCaffrey TA, Livingstone MB (2013) Associations of dietary glycaemic index and glycaemic load with food and nutrient intake and general and central obesity in British adults. Br J Nutr 9:1-11

25. Rossi M, Bosetti C, Talamini R et al (2010) Glycemic index and glycemic load in relation to body mass index and waist to hip ratio. Eur J Nutr 49:459-464

26. Mendez MA, Covas MI, Marrugat J et al (2009) Glycemic load, glycemic index, and body mass index in Spanish adults. Am J Clin Nutr 89:316-322

27. Ford ES, Liu S (2001) Glycemic index and serum high-density lipoprotein cholesterol concentration among US adults. Arch Intern Med 161:572-576

28. Denova-Gutiérrez E, Huitrón-Bravo G, Talavera JO et al (2010) Dietary glycemic index, dietary glycemic load, blood lipids, and coronary heart disease. J Nutr Metab. https://doi. org/10.1155/2010/170680

29. Levitan EB, Cook NR, Stampfer MJ et al (2008) Dietary glycemic index, dietary glycemic load, blood lipids, and C-reactive protein. Metabolism 57:437-443

30. Liu S, Manson JE, Stampfer MJ et al (2001) Dietary glycemic load assessed by food-frequency questionnaire in relation to plasma high-density lipoprotein cholesterol and fasting plasma triacylglycerols in postmenopausal women. Am J Clin Nutr 73:560-566

31. Lorenz K (2003) Rye bread: fermentation processes ABD products in the United States. In: Kulp K, Lorenz K (eds) Handbook of dough fermentations. Marcel Dekker Inc., New York, pp 159-191

32. Amano Y, Kawakubo K, Lee JS et al (2004) Correlation between dietary glycemic index and cardiovascular disease risk factors among Japanese women. Eur J Clin Nutr 58:1472-1478

33. Rosén LA, Östman EM, Shewry PR et al (2011) Postprandial glycemia, insulinemia, and satiety responses in healthy subjects after whole grain rye bread made from different rye varieties. 1 . J Agric Food Chem 59:12139-12148

34. Sarwar N, Gao P, Seshasai SR et al (2010) Diabetes mellitus, fasting blood glucose concentration, and risk of vascular disease: a collaborative meta-analysis of 102 prospective studies. The Emerging Risk Factors Collaboration. Lancet 375:2215-2222 
35. Sieri S, Krogh V, Berrino F et al (2010) Dietary glycemic load and index and risk of coronary heart disease in a large italian cohort: the EPICOR study. Arch Intern Med 170:640-647

36. Hu Y, Block G, Norkus EP et al (2006) Relations of glycemic index and glycemic load with plasma oxidative stress markers. Am J Clin Nutr 84:70-76

37. Arcidiacono B, Iiritano S, Nocera A et al (2012) Insulin resistance and cancer risk: an overview of the pathogenetic mechanisms. Exp Diabetes Res. https://doi.org/10.1155/2012/789174

38. Hu J, la Vecchia C, Augustin LS et al (2013) Glycemic index, glycemic load and cancer risk. Ann Oncol 24:245-251

39. Pereira MA, O'Reilly E, Augustsson K et al (2004) Dietary fiber and risk of coronary heart disease: a pooled analysis of cohort studies. Arch Intern Med 164:370-376

40. Schulze MB, Schulz M, Heidemann C et al (2007) Fiber and magnesium intake and incidence of type 2 diabetes: a prospective study and meta-analysis. Arch Intern Med 167:956-965

41. Koh-Banerjee P, Franz M, Sampson L et al (2004) Changes in whole-grain, bran, and cereal fiber consumption in relation to 8-y weight gain among men. Am J Clin Nutr 80:1237-1245

42. Aune D, Chan DS, Lau R et al (2011) Dietary fibre, whole grains, and risk of colorectal cancer: systematic review and dose-response meta-analysis of prospective studies. Br Med J 343:d6617

43. Esposito K, Nappo F, Giugliano F et al (2003) Meal modulation of circulating interleukin 18 and adiponectin concentrations in healthy subjects and in patients with type 2 diabetes mellitus. Am J Clin Nutr 78:1135-1140

44. Chuang SC, Vermeulen R, Sharabiani MT et al (2011) The intake of grain fibers modulates cytokine levels in blood. Biomarkers $16: 504-510$
45. Weickert MO, Möhlig M, Schöfl C et al (2006) Cereal fiber improves whole-body insulin sensitivity in overweight and obese women. Diabetes Care 29:775-780

46. Gil A, Ortega RM, Maldonado J (2011) Wholegrain cereals and bread: a duet of the Mediterranean diet for the prevention of chronic diseases. Public Health Nutr 14:2316-2322

47. Aune D, Norat T, Romundstad P et al (2013) Whole grain and refined grain consumption and the risk of type 2 diabetes: a systematic review and dose-response meta-analysis of cohort studies. Eur J Epidemiol 28:845-858

48. Fardet A (2010) New hypotheses for the health-protective mechanisms of whole-grain cereals: what is beyond fibre? Nutr Res Rev 23:65-134

49. Costabile A, Klinder A, Fava F et al (2008) Whole-grain wheat breakfast cereal has a prebiotic effect on the human gut microbiota: a double-blind, placebo-controlled, crossover study. Br J Nutr 99:110-120

50. Slavin J (2013) Fiber and prebiotics: mechanisms and health benefits. Nutrients 5:1417-1435

51. Nakamura YK, Omaye ST (2012) Metabolic diseases and pro- and prebiotics: mechanistic insights. Nutr Metab 9:60

Publisher's Note Springer Nature remains neutral with regard to jurisdictional claims in published maps and institutional affiliations. 